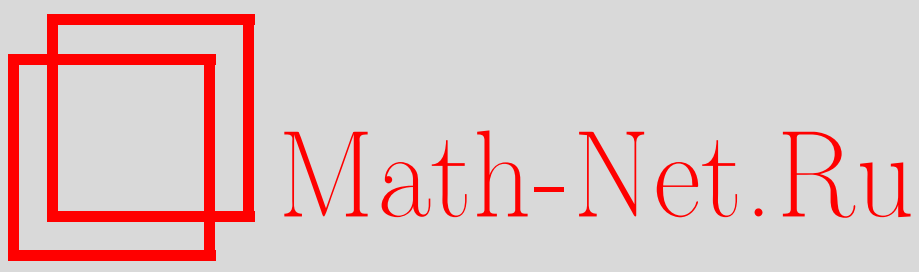

U. Kh. Mamadaliev, B. A. Omirov, Cohomologically rigid solvable leibniz algebras with nilradical of arbitrary characteristic sequence, Sibirsk. Mat. Zh., 2020, Volume 61, Number 3, 641-653

DOI: https://doi.org/10.33048/smzh.2020.61.312

Use of the all-Russian mathematical portal Math-Net.Ru implies that you have read and agreed to these terms of use http://www . mathnet.ru/eng/agreement

Download details:

IP: 34.227 .88 .159

April 26, 2023, 14:50:58

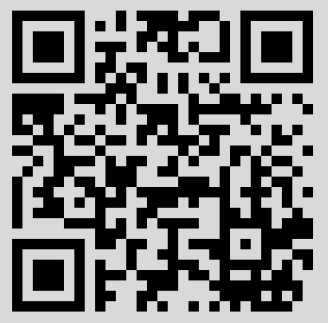


Сибирский математический журнал Май-июнь, 2020. Том 61, № 3

УДК 512.554

\title{
КОГОМОЛОГИЧЕСКИ ЖЕСТКИЕ РАЗРЕШИМЫЕ АЛГЕБРЫ ЛЕЙБНИЦА С НИЛЬРАДИКАЛОМ ПРОИЗВОЛЬНОЙ ХАРАКТЕРИСТИЧЕСКОЙ ПОСЛЕДОВАТЕЛЬНОСТИ
}

\author{
У. Х. Мамадалиев, Б. А. Омиров
}

\begin{abstract}
Аннотация. Приводится описание $(n+s)$-мерных разрешимых алгебр Лейбница с нильрадикалом, имеющим характеристическую последовательность, равную $\left(m_{1}, \ldots, m_{s}\right)$, где $m_{1}+\cdots+m_{s}=n$. Доказываются совершенность и когомологическая жесткость такой алгебры.
\end{abstract}

DOI 10.33048/smzh.2020.61.312

Ключевые слова: алгебра Лейбница, разрешимая алгебра, нильрадикал, жесткая алгебра, вторая группа когомологий.

Посвящается 80-летию академика Юрия Леонидовича Ершова

\section{$\S 1$. Введение}

Алгебры Лейбница были впервые введены в 1965 г. А. Блохом [1] под названием «D-алгебры». Данные алгебры появились снова в 1993 г. в работе Лоде [2], где за ними закрепилось нынешнее название «алгебры Лейбница». Алгебры Лейбница являются настолько естественным обобщением алгебр Ли, что многие свойства, справедливые для алгебр Ли, продолжаются на случай алгебр Лейбница.

Так же, как и другие конечномерные алгебры, которые задаются тождествами, алгебры Лейбница заданной размерности образуют алгебраическое многообразие. Ввиду того, что любое алгебраическое многообразие представляется в виде объединения конечного числа неприводимых компонент, а они, в свою очередь, описываются открытыми подмножествами, важным при описании многообразий конечномерных алгебр является описание алгебр, орбиты которых под действием линейной группы представляют собой открытые множества. Алгебры с такими орбитами называются жесткими алгебрами. Таким образом, замыкания орбит жестких алгебр дают неприводимые компоненты многообразия. Достаточным условием жесткости алгебр является тривиальность 2-й группы когомологий [3]. Отметим, что вычисление 2-х групп когомологий связано с довольно большими трудностями. Для случая алгебр Ли Хохшильд и Серр доказали теорему, которая значительно упрощает вычисление групп когомологий [4]. Для не лиевых алгебр Лейбница аналог такой теоремы, к сожалению, на данный момент отсутствует, поэтому в каждом отдельном случае приходится использовать структуру алгебры.

(c) 2020 Мамадалиев У. Х., Омиров Б. А. 
В данной работе приведена классификация разрешимых алгебр Лейбница с нильрадикалом, который имеет характеристическую последовательность, равную $\left(m_{1}, \ldots, m_{s}\right)$, при условии, что размерность дополняющего пространства нильрадикала имеет максимальное значение. Кроме того, доказываются совершенность и когомологическая жесткость таких алгебр Лейбница. Отметим, что данные алгебры являются лейбницевым аналогом алгебр Ли, рассмотренных в [5].

\section{§ 2. Предварительные сведения}

Приведем основные понятия и предварительные результаты.

ОПРЕДЕЛЕНиЕ 2.1. Алгебра $L$ над полем $F$ называется алгеброй Лейбнииа, если для любых $x, y, z \in L$ выполняется тождество

$$
[x,[y, z]]=[[x, y], z]-[[x, z], y]
$$

где $[\cdot, \cdot]$ - умножение в $L$.

Для произвольной алгебры Лейбница $L$ определим нижний центральный и производный ряды:

$$
L^{[1]}=L, \quad L^{[k+1]}=\left[L^{[k]}, L^{[k]}\right] ; \quad L^{1}=L, \quad L^{k+1}=\left[L^{k}, L^{1}\right], \quad k \geq 1 .
$$

ОПРедЕЛЕНИЕ 2.2. Алгебра Лейбница $L$ называется разрешимой (соответственно нильпотентной), если существует $s \in \mathbb{N}$ такое, что $L^{[s]}=0$ (соответственно $\left.L^{s}=0\right)$.

Максимальный нильпотентный идеал алгебры Лейбница $L$ называется нильрадикалом.

Отметим, что операторы правого умножения на элемент алгебры (т. е. $\left.\mathscr{R}_{x}(y)=[x, y]\right)$ являются дифференцированиями, которые будем называть внутренними.

ОПРЕДЕЛЕНИЕ 2.3. Алгебра Лейбница $L$ называется совершенной, если ее центр тривиальный и любое дифференцирование алгебры является внутренним.

Для более детального ознакомления с группой когомологий алгебр Лейбница отошлем читателя к работе [2]. Напомним только, что 2-коцикл $\varphi \in$ $Z L^{2}(L, M)$ и 2-кограница $f \in B L^{2}(L, M)$ алгебры Лейбница $L$ с коэффициентами в модуле $M$ определяются следующим образом:

$$
\begin{gathered}
\left(d^{2} \varphi\right)(a, b, c)=[a, \varphi(b, c)]-[\varphi(a, b), c]+[\varphi(a, c), b] \\
+\varphi(a,[b, c])-\varphi([a, b], c)+\varphi([a, c], b)=0, \\
f(a, b)=[g(a), b]+[a, g(b)]-g([a, b])
\end{gathered}
$$

для некоторого линейного отображения $g$. При этом

$$
H L^{2}(L, M)=Z L^{2}(L, M) / B L^{2}(L, M) .
$$

Определим действие группы $G L_{n}(F)$ на множестве всех $n$-мерных алгебр Лейбница $\operatorname{Leib}_{n}(F)$ следующим образом:

$$
(g * \lambda)(x, y):=g\left(\lambda\left(g^{-1}(x), g^{-1}(y)\right)\right), \quad g \in G L_{n}(F), \lambda \in \operatorname{Leib}_{n}(F) .
$$

Через $\operatorname{Orb}(L)$ обозначим орбиту алгебры $L$ при этом действии. Напомним, что алгебра $L$ называется жесткой, если $\operatorname{Orb}(L)$ - открытое множество в топологии Зариского. 
Существуют различные критерии жесткости, выражающие это исходно топологическое понятие в других терминах. Наиболее важным в этой связи является достаточный критерий жесткости, доказанный Балавоином [3]. А именно, тривиальность второй группы когомологий с коэффициентами в модуле, заданном регулярным представлением, влечет жесткость алгебры.

ОПРедЕЛЕниЕ 2.4. Алгебра Лейбница $L$ называется когомологически жесткой, если

$$
H L^{2}(L, L)=0 .
$$

Пусть $L-$ нильпотентная алгебра Лейбница, и пусть $x \in L \backslash L^{2}$. Для нильпотентного оператора правого умножения $\mathscr{R}_{x}$ рассмотрим убывающую последовательность $C(x)=\left(m_{1}, \ldots, m_{s}\right)$, состоящую из размеров жордановых клеток оператора $\mathscr{R}_{x}$. На множестве таких последовательностей определим лексикографический порядок.

ОПРЕДЕЛЕНИЕ 2.5. Последовательность

$$
C(L)=\max _{x \in L \backslash L, L]} C(x)
$$

называется характеристической последовательностъю алгебры $L$.

\section{§ 3. Основные результаты}

Рассмотрим нильпотентную алгебру Лейбница с характеристической последовательностью $\left(m_{1}, \ldots, m_{s}\right)$, где $m_{1} \geq \cdots \geq m_{s} \geq 1$, и таблицей умножения

$$
\left[e_{i}^{t}, e_{1}^{1}\right]=e_{i+1}^{t}, \quad 1 \leq t \leq s, 1 \leq i \leq m_{t}-1 .
$$

Обозначим эту алгебру через $N_{m_{1}, \ldots, m_{s}}$. Для разрешимых алгебр Лейбница с нильрадикалом $N_{m_{1}, \ldots, m_{s}}$ и размерностью дополняющего пространства, равной $s$, будем использовать обозначение $R\left(N_{m_{1}, \ldots, m_{s}}, s\right)$.

Теорема 3.1. Произвольная разрешимая алгебра Лейбница $R\left(N_{m_{1}, \ldots, m_{s}}, s\right)$ изоморфна следующей алгебре:

$$
\begin{cases}{\left[e_{i}^{t}, e_{1}^{1}\right]=e_{i+1}^{t},} & 1 \leq t \leq s, 1 \leq i \leq m_{t}-1, \\ {\left[e_{i}^{1}, x_{1}\right]=i e_{i}^{1},} & 1 \leq i \leq m_{1}, \\ {\left[e_{i}^{t}, x_{1}\right]=(i-1) e_{i}^{t},} & 2 \leq t \leq s, 2 \leq i \leq m_{t}, \\ {\left[e_{i}^{t}, x_{t}\right]=e_{i}^{t},} & 2 \leq t \leq s, 1 \leq i \leq m_{t}, \\ {\left[x_{1}, e_{1}^{1}\right]=-e_{1}^{1},} & \end{cases}
$$

где $\left\{x_{1}, \ldots x_{s}\right\}$ - базис дополняющего векторного пространства.

Доказательство теоремы проводится методами, аналогичными доказательству теоремы 3.2 из [6].

Опишем пространство дифференцирований алгебры $R\left(N_{m_{1}, \ldots, m_{s}}, s\right)$.

Предложение 3.2. Следующие линейные преобразования образуют базис пространства $\operatorname{Der}\left(R\left(N_{m_{1}, \ldots, m_{s}}, s\right)\right)$ :

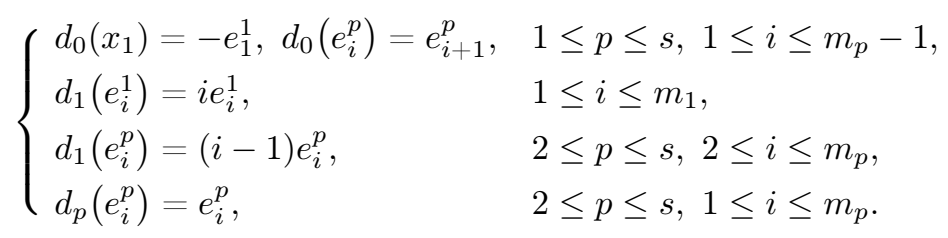


ДокАзАтЕльство. Пусть $d$ - дифференцирование алгебры $R\left(N_{m_{1}, \ldots, m_{s}}, s\right)$. Положим

$d\left(e_{1}^{p}\right)=\sum_{t=1}^{s} \sum_{i=1}^{m_{t}} \alpha_{t, i}^{p} e_{i}^{t}+\sum_{i=1}^{s} \beta_{1, i}^{p} x_{i}, \quad d\left(x_{p}\right)=\sum_{t=1}^{s} \sum_{i=1}^{m_{t}} \gamma_{t, i}^{p} e_{i}^{t}+\sum_{i=1}^{s} \beta_{2, i}^{p} x_{i}, \quad 1 \leq p \leq s$.

Из равенства $d\left(\left[e_{1}^{1}, x_{1}\right]\right)=\left[d\left(e_{1}^{1}\right), x_{1}\right]+\left[e_{1}^{1}, d\left(x_{1}\right)\right]$ получим следующие ограничения:

$$
\begin{cases}\gamma_{1,1}^{1}=-\alpha_{1,2}^{1}, \beta_{2,1}^{1}=0, & \\ \alpha_{1, i}^{1}=0, & 3 \leq i \leq m_{1} \\ \alpha_{t, i}^{1}=0, & 2 \leq t \leq s, i=1,3 \leq i \leq m_{t} \\ \beta_{1, i}^{1}=0, & 1 \leq i \leq s .\end{cases}
$$

Для $2 \leq p \leq s$, рассмотрев равенство $d\left(\left[e_{1}^{p}, x_{1}\right]\right)=\left[d\left(e_{1}^{p}\right), x_{1}\right]+\left[e_{1}^{p}, d\left(x_{1}\right)\right]$, получим

$$
\begin{cases}\alpha_{p, 2}^{p}=\alpha_{1,2}^{1}, & \\ \alpha_{1, i}^{p}=0, & 1 \leq i \leq m_{1} \\ \alpha_{t, i}^{p}=0, & 2 \leq t \leq s, 2 \leq i \leq m_{t} \\ \beta_{2, p}^{1}=0 & \end{cases}
$$

Для $1 \leq p \leq s$ из равенства $0=d\left(\left[x_{p}, x_{1}\right]\right)=\left[d\left(x_{p}\right), x_{1}\right]+\left[x_{p}, d\left(x_{1}\right)\right]$ вытекает

$$
\begin{cases}\gamma_{t, i}^{p}=0, & 1 \leq t \leq s, 2 \leq i \leq m_{t}, 1 \leq p \leq s \\ \gamma_{1,1}^{p}=0, & 2 \leq p \leq s\end{cases}
$$

Равенство $d\left(\left[e_{1}^{1}, x_{p}\right]\right)=\left[d\left(e_{1}^{1}\right), x_{p}\right]+\left[e_{1}^{1}, d\left(x_{p}\right)\right]$ для $2 \leq p \leq s$ влечет

Следовательно,

$$
\alpha_{p, 2}^{1}=\beta_{2,1}^{p}=0, \quad 2 \leq p \leq s .
$$

$$
d\left(e_{1}^{1}\right)=\alpha_{1,1}^{1} e_{1}^{1}+\alpha_{1,2}^{1} e_{2}^{1}, \quad d\left(x_{p}\right)=\sum_{t=2}^{s} \gamma_{t, 1}^{p} e_{1}^{p}+\sum_{i=2}^{s} \beta_{2, i}^{p} x_{i}, \quad 2 \leq p \leq s .
$$

Из равенства $0=d\left(\left[x_{p}, e_{1}^{1}\right]\right)=\left[d\left(x_{p}\right), e_{1}^{1}\right]+\left[x_{p}, d\left(e_{1}^{1}\right)\right]$ для $1 \leq p \leq s$ получим

$$
\gamma_{t, 1}^{p}=0, \quad 2 \leq t \leq s
$$

Из соотношения $d\left(\left[e_{1}^{p}, x_{j}\right]\right)=\left[d\left(e_{1}^{p}\right), x_{j}\right]+\left[e_{1}^{p}, d\left(x_{j}\right)\right]$ для $2 \leq p, j \leq s$ имеем

Следовательно,

$$
\left\{\begin{array}{l}
\alpha_{j, 1}^{p}=0, \quad 2 \leq p, j \leq s, p \neq j \\
\beta_{t, j}^{p}=0, \quad 2 \leq p \leq s, 1 \leq t \leq 2,1 \leq j \leq s
\end{array}\right.
$$

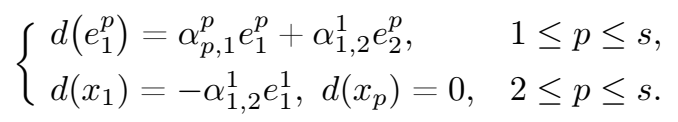

Из цепочки равенств

$$
\left.d\left(e_{i}^{p}\right)=d\left(\left[e_{i-1}^{p}, e_{1}^{1}\right]\right)=\left[d\left(e_{i-1}^{p}\right), e_{1}^{1}\right]\right)+\left[e_{i-1}^{p}, d\left(e_{1}^{1}\right)\right], \quad 1 \leq p \leq s, 2 \leq i \leq m_{p},
$$

и полученных выше ограничений нетрудно установить, что

$$
\begin{aligned}
d\left(e_{i}^{1}\right) & =i \alpha_{1,1}^{1} e_{i}^{1}+\alpha_{1,2}^{1} e_{i+1}^{1}, \quad 1 \leq i \leq m_{1}-1, \\
d\left(e_{m_{1}}^{1}\right) & =m_{1} \alpha_{1,1}^{1} e_{m_{1}}^{1}, \\
d\left(e_{i}^{p}\right) & =\left((i-1) \alpha_{1,1}^{1}+\alpha_{p, 1}^{p}\right) e_{i}^{p}+\alpha_{1,2}^{1} e_{i+1}^{p}, \quad 2 \leq p \leq s, 1 \leq i \leq m_{p}-1, \\
d\left(e_{i}^{p}\right) & =\left(\left(m_{p}-1\right) \alpha_{1,1}^{1}+\alpha_{p, 1}^{p}\right) e_{m_{p}}^{p}, \quad 2 \leq p \leq s .
\end{aligned}
$$

Из теоремы 3.1 и предложения 3.2 вытекает следующий результат. 
Следствие 3.3. Алгебра Лейбница $R\left(N_{m_{1}, \ldots, m_{s}}, s\right)$ является совершенной алгеброй.

Пусть $L_{2}$ - идеал разрешимой алгебры Лейбница $R$ такой, что $L_{1} \simeq R / L_{2}-$ подалгебра алгебры $R$. Обозначим через $\overline{Z L^{2}\left(L_{1}, L_{1}\right)}, \overline{B L^{2}\left(L_{1}, L_{1}\right)}$ дополняющие пространства пространств $Z L^{2}\left(L_{1}, L_{1}\right), B L^{2}\left(L_{1}, L_{1}\right)$ до пространств $Z L^{2}(R, R)$, $B L^{2}(R, R)$ соответственно.

Перейдем к доказательству основного результата работы, а именно докажем, что $H L^{2}\left(R\left(N_{m_{1}, \ldots, m_{s}}, s\right), R\left(N_{m_{1}, \ldots, m_{s}}, s\right)\right)=0$. Будем доказывать данный результат индукцией по $s$.

При $s=1$ алгебра $R\left(N_{m_{1}}, 1\right)$ является разрешимой алгеброй с нуль-филиформным нильрадикалом. Из [7] известно, что $H L^{2}\left(R\left(N_{m_{1}}, 1\right), R\left(N_{m_{1}}, 1\right)\right)=0$. Поэтому основание индукции имеется.

Предположим, что $H L^{2}\left(R\left(N_{m_{1}, \ldots, m_{k}}, k\right), R\left(N_{m_{1}, \ldots, m_{k}}, k\right)\right)=0$ для всех $k \leq$ $s-1$. Ввиду того, что доказательство для $s$ очень громоздко, проведем доказательство для $s=2$, из которого можно видеть, как будет реализовываться индукционный переход для $s$.

При $s=2$ алгебра $R\left(N_{m_{1}, m_{2}}, 2\right)$ имеет таблицу умножения

$$
R\left(N_{m_{1}, m_{2}}, 2\right): \begin{cases}{\left[e_{i}, e_{1}\right]=e_{i+1},} & 1 \leq i \leq m_{1}-1 \\ {\left[f_{i}, e_{1}\right]=f_{i+1},} & 1 \leq i \leq m_{2}-1 \\ {\left[e_{i}, x_{1}\right]=i e_{i},} & 1 \leq i \leq m_{1} \\ {\left[f_{i}, x_{1}\right]=(i-1) f_{i},} & 1 \leq i \leq m_{2} \\ {\left[f_{i}, x_{2}\right]=f_{i},} & 1 \leq i \leq m_{2}, \\ {\left[x_{1}, e_{1}\right]=-e_{1},} & \end{cases}
$$

где $\left\{e_{1}, \ldots, e_{m_{1}}, f_{1}, f_{2}, \ldots, f_{m_{2}}\right\}$ - базис нильрадикала, а $\left\{x_{1}, x_{2}\right\}$ - базис дополняющего векторного пространства.

Доказательство тривиальности $H L^{2}\left(R\left(N_{m_{1}, m_{2}}, 2\right), R\left(N_{m_{1}, m_{2}}, 2\right)\right)$ будем проводить индукцией по $m_{2}$.

При $m_{2}=1$ алгебра $R\left(N_{m_{1}, 1}, 2\right)$ имеет следующую таблицу умножения:

$$
R\left(N_{m_{1}, 1}, 2\right):\left\{\begin{array}{l}
{\left[e_{i}, e_{1}\right]=e_{i+1}, \quad 1 \leq i \leq m_{1}-1} \\
{\left[e_{i}, x_{1}\right]=i e_{i}, \quad 1 \leq i \leq m_{1}} \\
{\left[f_{1}, x_{2}\right]=f_{1}} \\
{\left[x_{1}, e_{1}\right]=-e_{1}}
\end{array}\right.
$$

Из данной таблицы умножения видно, что $J=\operatorname{span}\left\{f_{1}, x_{2}\right\}$ является идеалом и фактор-алгебра $R\left(N_{m_{1}, 1}, 2\right) / J \simeq R\left(N_{m_{1}}, 1\right)$ изоморфно вкладывается в алгебру Лейбница $R\left(N_{m_{1}, 1}, 2\right)$, причем тривиальность 2 -группы когомологий алгебры $R\left(N_{m_{1}}, 1\right)$ известна из [7].

Так как тривиальность второй группы когомологий алгебры $R\left(N_{m_{1}, 1}, 2\right)$ равносильна равенству

$$
\operatorname{dim} \overline{Z L^{2}\left(R\left(N_{m_{1}}, 1\right), R\left(N_{m_{1}}, 1\right)\right)}=\operatorname{dim} \overline{B L^{2}\left(R\left(N_{m_{1}}, 1\right), R\left(N_{m_{1}}, 1\right)\right)},
$$

будем его доказывать в следуюших двух предложениях и их следствиях.

Предложение 3.4. Следующие 2-коциклы образуют базис пространства: 


$$
\begin{array}{lll}
\overline{Z L^{2}\left(R\left(N_{m_{1}}, 1\right), R\left(N_{m_{1}}, 1\right)\right)}: & \\
\varphi_{i, 1}\left(e_{i}, e_{1}\right)=f_{1}, \quad \varphi_{i, 1}\left(e_{i+1}, x_{1}\right)=(i+1) f_{1}, & \varphi_{i, 1}\left(e_{i+1}, x_{2}\right)=-f_{1}, 1 \leq i \leq m_{1}-1, \\
\varphi_{i, 2}\left(e_{i}, e_{1}\right)=x_{2}, \quad \varphi_{i, 2}\left(e_{i+1}, x_{1}\right)=(i+1) x_{2}, \varphi_{i, 2}\left(f_{1}, e_{i+1}\right)=f_{1}, & 1 \leq i \leq m_{1}-1, \\
\psi_{1}\left(f_{1}, e_{1}\right)=e_{1}, \quad \psi_{1}\left(f_{1}, x_{2}\right)=x_{1}, & \psi_{1}\left(e_{i}, f_{1}\right)=-i e_{i}, & 1 \leq i \leq m_{1}, \\
\psi_{2}\left(x_{1}, f_{1}\right)=-e_{1}, \psi_{2}\left(e_{i}, f_{1}\right)=e_{i+1}, & \psi_{2,2}\left(x_{2}, x_{2}\right)=f_{1}, \quad 1 \leq i \leq m_{1}, \\
\psi_{i}\left(f_{1}, e_{1}\right)=e_{i}, \quad \psi_{i}\left(f_{1}, x_{1}\right)=(i-1) e_{i-1}, & \psi_{i}\left(f_{1}, x_{2}\right)=-e_{i-1}, \quad 2 \leq i \leq m_{1}-1, \\
\psi_{1,1}\left(f_{1}, e_{1}\right)=f_{1}, \quad \psi_{1,1}\left(x_{1}, e_{1}\right)=x_{2}, & \psi_{1,1}\left(e_{1}, x_{1}\right)=-x_{2}, & \\
\xi_{1}\left(x_{2}, e_{1}\right)=e_{1}, \quad \xi_{1}\left(e_{1}, x_{2}\right)=-e_{1}, & \xi_{1}\left(e_{i}, x_{2}\right)=-i e_{i}, & 2 \leq i \leq m_{1}, \\
\xi_{2}\left(e_{1}, x_{2}\right)=e_{2}, \quad \xi_{2}\left(e_{i}, x_{2}\right)=e_{i+1}, & \xi_{2}\left(x_{1}, x_{2}\right)=-e_{1}, & 2 \leq i \leq m_{1}, \\
\xi_{i}\left(x_{2}, e_{1}\right)=e_{i}, \quad \xi_{i}\left(x_{2}, x_{1}\right)=(i-1) e_{i-1}, & \eta\left(f_{1}, x_{2}\right)=f_{1}, & 2 \leq i \leq m_{1}, \\
\phi_{1}\left(x_{1}, e_{1}\right)=f_{1}, \quad \phi_{1}\left(e_{1}, x_{2}\right)=f_{1}, & \phi_{2}\left(x_{1}, x_{2}\right)=f_{1}, & \\
\phi_{3}\left(f_{1}, x_{2}\right)=-x_{2}, \phi_{3}\left(f_{1}, f_{1}\right)=f_{1}, & \phi_{4}\left(x_{2}, x_{1}\right)=e_{m_{1}}, & \\
\chi_{1}\left(f_{1}, x_{1}\right)=e_{m_{1}}, \chi_{1}\left(f_{1}, x_{2}\right)=-\frac{1}{m_{1}} e_{m_{1}}, & \chi_{2}\left(f_{1}, x_{1}\right)=f_{1} . &
\end{array}
$$

ДокАЗАтЕЛЬСтво. Пусть $\varphi \in \overline{Z L^{2}\left(R\left(N_{m_{1}}, 1\right), R\left(N_{m_{1}}, 1\right)\right)}$, тогда нетрудно видеть, что $\varphi(a, b) \in J$ для любых $a, b \in R\left(N_{m_{1}}, 1\right)$.

\section{Положим}

$$
\begin{gathered}
\varphi\left(e_{i}, e_{1}\right)=\xi_{i, 1} f_{1}+\xi_{i, 2} x_{2}, \quad 1 \leq i \leq m_{1}, \\
\varphi\left(x_{1}, e_{1}\right)=\xi_{1,1}^{2} f_{1}+\xi_{1,2}^{2} x_{2}, \varphi\left(x_{1}, x_{1}\right)=\nu_{1} f_{1}+\nu_{2} x_{2}, \\
\varphi\left(f_{1}, e_{1}\right)=\sum_{i=1}^{m_{1}} \alpha_{1, i}^{1} e_{i}+\alpha_{1}^{1} f_{1}+\alpha_{3,1}^{1} x_{1}+\alpha_{3,2}^{1} x_{2}, \\
\varphi\left(e_{1}, f_{1}\right)=\sum_{i=1}^{m_{1}} \alpha_{1, i}^{2} e_{i}+\alpha_{1}^{2} f_{1}+\alpha_{3,1}^{2} x_{1}+\alpha_{3,2}^{2} x_{2}, \\
\varphi\left(e_{1}, x_{2}\right)=\sum_{i=1}^{m_{1}} \delta_{1, i} e_{i}+\delta_{1} f_{1}+\delta_{3,1}^{1} x_{1}+\delta_{3,2}^{1} x_{2}, \\
\varphi\left(x_{2}, e_{1}\right)=\sum_{i=1}^{m_{1}} \delta_{2, i} e_{i}+\delta_{2} f_{1}+\delta_{3,1}^{2} x_{1}+\delta_{3,2}^{2} x_{2}, \\
\varphi\left(f_{1}, x_{1}\right)=\sum_{i=1}^{m_{1}} \beta_{1, i} e_{i}+\beta_{1} f_{1}+\beta_{1,1}^{3} x_{1}+\beta_{1,2}^{3} x_{2}, \\
\varphi\left(x_{1}, f_{1}\right)=\sum_{i=1}^{m_{1}} \beta_{2, i} e_{i}+\beta_{2} f_{1}+\beta_{2,1}^{3} x_{1}+\beta_{2,2}^{3} x_{2}, \\
\varphi\left(f_{1}, x_{2}\right)=\sum_{i=1}^{m_{1}} \beta_{1,2}^{i} e_{i}+\beta_{2}^{2} f_{1}+\beta_{1,2}^{1,1} x_{1}+\beta_{1,2}^{1,2} x_{2}, \\
\varphi\left(x_{2}, f_{1}\right)=\sum_{i=1}^{m_{1}} \beta_{2,1}^{i} e_{i}+\beta_{2,1}^{2,2} f_{1}+\beta_{2,1}^{1,1} x_{1}+\beta_{2,1}^{1,2} x_{2}, \\
\left.f_{1}, f_{1}\right)=\sum_{i=1}^{m_{1}} \alpha_{2, i}^{2} e_{i}+\alpha_{2,2} f_{1}+\alpha_{2,1}^{3} x_{1}+\alpha_{2,2}^{3} x_{2}, \gamma_{2,1}^{1,2} f_{1}+\gamma_{3,1}^{1,2} x_{1}+\gamma_{3,2}^{1,2} x_{2},
\end{gathered}
$$




$$
\begin{aligned}
& \varphi\left(x_{2}, x_{1}\right)=\sum_{i=1}^{m_{1}} \gamma_{2,1}^{i} e_{i}+\gamma_{2,1}^{2,1} f_{1}+\gamma_{3,1}^{2,1} x_{1}+\gamma_{3,2}^{2,1} x_{2}, \\
& \varphi\left(x_{2}, x_{2}\right)=\sum_{i=1}^{m_{1}} \gamma_{2,2}^{i} e_{i}+\gamma_{2,1}^{2,2} f_{1}+\gamma_{3,1}^{2,2} x_{1}+\gamma_{3,2}^{2,2} x_{2} .
\end{aligned}
$$

Рассмотрев равенства $\left(d^{2} \varphi\right)\left(e_{i}, e_{1}, x_{1}\right)=\left(d^{2} \varphi\right)\left(x_{1}, e_{1}, x_{1}\right)=0$, получим

$$
\varphi\left(e_{i}, x_{1}\right)=i \varphi\left(e_{i-1}, e_{1}\right), 2 \leq i \leq m_{1}, \quad \varphi\left(e_{1}, x_{1}\right)=-\varphi\left(x_{1}, e_{1}\right) .
$$

Подставляя в тождество $\left(d^{2} \varphi\right)(x, y, z)=0$ всевозможные базисные элементы $x, y, z \in R\left(N_{m_{1}, 1}, 2\right)$ и учитывая, что $e_{2}, \ldots, e_{m_{1}}, f_{1} \in \operatorname{Ann}_{r}\left(R\left(N_{m_{1}, 1}, 2\right)\right)$, получаем ограничения на параметры, задающие $\varphi$, где слева в импликации указаны тройки, справа - ограничения:

$$
\begin{aligned}
\left\{f_{1}, e_{1}, x_{2}\right\} \Rightarrow & \beta_{1,2}^{1,1}=\alpha_{1,1}^{1}, \beta_{1,2}^{i}=-\alpha_{1, i+1}^{1}, 1 \leq i \leq m_{1}-1, \\
& \delta_{3,2}^{1}=\alpha_{3,1}^{1}=\alpha_{3,2}^{1}=0, \\
\left\{f_{1}, e_{1}, x_{1}\right\} \Rightarrow & \beta_{1, i}=i \alpha_{1, i+1}^{1}, \xi_{1,2}^{2}=\alpha_{1}^{1}, \quad 1 \leq i \leq m_{1}-1, \\
\left\{x_{2}, e_{1}, x_{2}\right\} \Rightarrow & \gamma_{2,2}^{i}=\gamma_{3,1}^{2,2}=\delta_{2}=0, \quad 1 \leq i \leq m_{1}-1, \\
\left\{f_{1}, x_{2}, x_{2}\right\} \Rightarrow & \gamma_{3,2}^{2,2}=0 \\
\left\{x_{2}, x_{2}, x_{1}\right\} \Rightarrow & \gamma_{2,2}^{m_{1}}=\gamma_{2,1}^{2,1}=0, \\
\left\{x_{2}, x_{1}, e_{1}\right\} \Rightarrow & \gamma_{2,1}^{i}=i \delta_{2, i+1}, \quad \gamma_{3,1}^{2,1}=\delta_{3,1}^{2}=\delta_{3,2}^{2}=0, \quad 1 \leq i \leq m_{1}-1, \\
\left\{f_{1}, x_{1}, e_{1}\right\} \Rightarrow & \beta_{1,1}^{3}=0, \\
\left\{f_{1}, x_{2}, x_{1}\right\} \Rightarrow & \gamma_{3,2}^{2,1}=\beta_{1,2}^{3}=0, \beta_{1,2}^{m_{1}}=-\frac{1}{m_{1}} \beta_{1, m_{1}}, \\
\left\{x_{1}, x_{2}, x_{1}\right\} \Rightarrow & \gamma_{1,2}^{i}=\nu_{1}=0, \quad \gamma_{1,2}^{1}=-\delta_{2,2}, \quad 2 \leq i \leq m_{1}, \\
\left\{f_{1}, x_{1}, x_{1}\right\} \Rightarrow & \nu_{2}=0, \\
\left\{e_{1}, x_{1}, x_{2}\right\} \Rightarrow & \delta_{1, i}=\gamma_{3,1}^{1,2}=\delta_{3,1}^{1}=\delta_{3,2}^{1}=0, \quad 3 \leq i \leq m_{1}, \\
& \delta_{1,2}=\delta_{2,2}, \delta_{1}=\xi_{1,1}^{2}, \\
\left\{f_{1}, x_{1}, x_{2}\right\} \Rightarrow & \gamma_{3,2}^{1,2}=0, \\
\left\{x_{1}, x_{2}, e_{1}\right\} \Rightarrow & \delta_{1,1}=-\delta_{2,1}, \\
\left\{x_{1}, f_{1}, x_{1}\right\} \Rightarrow & \beta_{2,1}=-\alpha_{1,2}^{1}, \beta_{2, i}=0, \quad 2 \leq i \leq m_{1}, \\
\left\{x_{1}, f_{1}, x_{2}\right\} \Rightarrow & \beta_{2,1}^{3}=\beta_{2,2}^{3}=0 \\
\left\{x_{2}, f_{1}, x_{2}\right\} \Rightarrow & \beta_{2,1}^{1,1}=\beta_{2,1}^{1,2}=\beta_{2,1}^{i}=0, \quad 1 \leq i \leq m_{1}, \\
\left\{x_{2}, x_{2}, f_{1}\right\} \Rightarrow & \beta_{2,1}^{2,2}=0, \\
\left\{x_{1}, x_{2}, f_{1}\right\} \Rightarrow & \beta_{2}=0, \\
\left\{f_{1}, f_{1}, x_{2}\right\} \Rightarrow & \beta_{1,2}^{1,2}=-\alpha_{2,2}, \quad \alpha_{2, i}^{2}=\alpha_{2,1}^{3}=\alpha_{2,2}^{3}=0, \quad 1 \leq i \leq m_{1}, \\
\left\{e_{1}, f_{1}, x_{1}\right\} \Rightarrow & \alpha_{1,2}^{2}=\alpha_{1,2}^{1}, \quad \alpha_{1, i}^{2}=\alpha_{1}^{2}=\alpha_{3,1}^{2}=\alpha_{3,2}^{2}=0, \quad 3 \leq i \leq m_{1}, \\
\left\{e_{1}, f_{1}, x_{2}\right\} \Rightarrow & \alpha_{1,1}^{2}=-\alpha_{1,1}^{1} . \\
P_{2} &
\end{aligned}
$$

Рассмотрев тождество $\left(d^{2} \varphi\right)(x, y, z)=0$ для следующих троек элементов:

$$
\left\{e_{i}, e_{1}, f_{1}\right\}, \quad\left\{e_{1}, e_{i}, e_{1}\right\}, \quad\left\{e_{1}, e_{1}, e_{i}\right\}, \quad\left\{e_{i}, e_{1}, e_{j}\right\}, \quad\left\{f_{1}, e_{i}, e_{1}\right\}
$$




$$
\left\{x_{1}, e_{i}, e_{1}\right\}, \quad\left\{e_{i}, e_{1}, x_{2}\right\}, \quad\left\{e_{m_{1}}, e_{1}, x_{1}\right\}, \quad\left\{x_{2}, e_{i}, e_{1}\right\}
$$

при $1 \leq i, j \leq m_{1}$, рекуррентным образом получим

$$
\begin{gathered}
\varphi\left(e_{i}, f_{1}\right)=-i \alpha_{1,1}^{1} e_{i}+\alpha_{1,2}^{1} e_{i+1}, \quad \varphi\left(f_{1}, e_{i}\right)=-\xi_{i-1,2} f_{1}, \quad 2 \leq i \leq m_{1}, \\
\varphi\left(e_{i}, x_{2}\right)=-i \delta_{2,1} e_{i}+\delta_{2,2} e_{i+1}-\xi_{i-1,1} f_{1}, \quad 2 \leq i \leq m_{1}, \\
\varphi\left(e_{m_{1}}, e_{1}\right)=\varphi\left(e_{i}, e_{j}\right)=\varphi\left(x_{1}, e_{j}\right)=\varphi\left(x_{2}, e_{j}\right)=0, \quad 1 \leq i \leq m_{1}, 2 \leq j \leq m_{1} .
\end{gathered}
$$

Таким образом, всякий элемент $\varphi \in \overline{Z L^{2}\left(R\left(N_{m_{1}}, 1\right), R\left(N_{m_{1}}, 1\right)\right)}$ имеет следующий вид:

$$
\begin{gathered}
\varphi\left(e_{i}, e_{1}\right)=\xi_{i, 1} f_{1}+\xi_{i, 2} x_{2}, \quad \varphi\left(f_{1}, e_{1}\right)=\sum_{i=1}^{m_{1}} \alpha_{1, i}^{1} e_{i}+\alpha_{1}^{1} f_{1}, \quad 1 \leq i \leq m_{1}-1, \\
\varphi\left(x_{1}, e_{1}\right)=\xi_{1,1}^{2} f_{1}+\alpha_{1}^{1} x_{2}, \quad \varphi\left(x_{2}, e_{1}\right)=\sum_{i=1}^{m_{1}} \delta_{2, i} e_{i}, \quad \varphi\left(x_{2}, x_{2}\right)=\gamma_{2,1}^{2,2} f_{1}, \\
\varphi\left(f_{1}, f_{1}\right)=\alpha_{2,2} f_{1}, \quad \varphi\left(f_{1}, x_{1}\right)=\sum_{i=1}^{m_{1}-1} i \alpha_{1, i+1}^{1} e_{i}+\beta_{1, m_{1}} e_{m_{1}}+\beta_{1} f_{1}, \\
\varphi\left(f_{1}, x_{2}\right)=-\sum_{i=1}^{m_{1}-1} \alpha_{1, i+1}^{1} e_{i}-\frac{1}{m_{1}} \beta_{1, m_{1}} e_{m_{1}}+\beta_{2}^{2} f_{1}+\alpha_{1,1}^{1} x_{1}-\alpha_{2,2} x_{2}, \\
\varphi\left(x_{1}, x_{2}\right)=-\delta_{2,2} e_{1}+\gamma_{2,1}^{1,2} f_{1}, \varphi\left(x_{2}, x_{1}\right)=\sum_{i=1}^{m_{1}-1} i \delta_{2, i+1} e_{i}+\gamma_{2,1}^{m_{1}} e_{m_{1}} \\
\varphi\left(e_{1}, x_{2}\right)=-\delta_{2,1} e_{1}+\delta_{2,2} e_{2}+\xi_{1,1}^{2} f_{1}, \quad \varphi\left(x_{1}, f_{1}\right)=-\alpha_{1,2}^{1} e_{1}, \\
\varphi\left(e_{i}, f_{1}\right)=-i \alpha_{1,1}^{1} e_{i}+\alpha_{1,2}^{1} e_{i+1}, \quad 1 \leq i \leq m_{1}, \\
\varphi\left(e_{1}, x_{1}\right)=-\varphi\left(x_{1}, e_{1}\right), \quad \varphi\left(e_{i}, x_{1}\right)=i \varphi\left(e_{i-1}, e_{1}\right), 2 \leq i \leq m_{1}, \\
\varphi\left(f_{1}, e_{i}\right)=-\xi_{i-1,2} f_{1}, \quad \varphi\left(e_{i}, x_{2}\right)=-i \delta_{2,1} e_{i}+\delta_{2,2} e_{i+1}-\xi_{i-1,1} f_{1}, 2 \leq i \leq m_{1}
\end{gathered}
$$

Следствие 3.5. $\operatorname{dim} \overline{Z L^{2}\left(R\left(N_{m_{1}}, 1\right), R\left(N_{m_{1}}, 1\right)\right)}=4 m_{1}+7$.

Аналогично предложению 3.4 доказывается

Предложение 3.6. Следующие 2-кограницы образуют базис пространства $\overline{B L^{2}\left(R\left(N_{m_{1}}, 1\right), R\left(N_{m_{1}}, 1\right)\right)}$ :

$$
\begin{array}{llll}
\widetilde{\varphi}_{1,1}\left(x_{1}, e_{1}\right)=f_{1}, & \widetilde{\varphi}_{i, 1}\left(e_{i}, x_{1}\right)=-i f_{1}, & \widetilde{\varphi}_{i, 1}\left(e_{i}, x_{2}\right)=f_{1}, & 1 \leq i \leq m_{1}, \\
\widetilde{\varphi}_{i, 2}\left(e_{i-1}, e_{1}\right)=-x_{2}, \widetilde{\varphi}_{i, 1}\left(e_{i-1}, e_{1}\right)=-f_{1}, \widetilde{\varphi}_{i, 2}\left(f_{1}, e_{i}\right)=f_{1}, & 2 \leq i \leq m_{1}, \\
\widetilde{\varphi}_{1,2}\left(f_{1}, e_{1}\right)=f_{1}, & \widetilde{\varphi}_{1,2}\left(x_{1}, e_{1}\right)=x_{2}, & \widetilde{\varphi}_{i, 2}\left(e_{i}, x_{1}\right)=-i x_{2}, 1 \leq i \leq m_{1}, \\
\tilde{\xi}_{1}\left(e_{i}, f_{1}\right)=e_{i+1}, & \tilde{\xi}_{i}\left(f_{1}, x_{2}\right)=-e_{i}, & \tilde{\xi}_{i}\left(f_{1}, x_{1}\right)=i e_{i}, & 1 \leq i \leq m_{1}, \\
\tilde{\xi}_{1}\left(x_{1}, f_{1}\right)=-e_{1}, & \widetilde{\xi}_{i}\left(f_{1}, e_{1}\right)=e_{i+1}, & & 1 \leq i \leq m_{1}-1, \\
\tilde{\xi}_{1,1}\left(f_{1}, x_{2}\right)=-x_{1}, & \widetilde{\xi}_{1,1}\left(f_{1}, e_{1}\right)=-e_{1}, & \tilde{\xi}_{1,1}\left(e_{i}, f_{1}\right)=i e_{i}, & 1 \leq i \leq m_{1}, \\
\widetilde{\xi}_{1,2}\left(f_{1}, x_{2}\right)=-x_{2}, & \widetilde{\xi}_{1,2}\left(f_{1}, f_{1}\right)=f_{1}, & \widetilde{\xi}_{1,3}\left(f_{1}, x_{2}\right)=f_{1}, & \\
\widetilde{\psi}_{1}\left(x_{1}, x_{2}\right)=-e_{1}, & \widetilde{\psi}_{1}\left(e_{i}, x_{2}\right)=e_{i+1}, & \widetilde{\psi}_{i}\left(x_{2}, e_{1}\right)=e_{i+1}, & 1 \leq i \leq m_{1}-1, \\
\widetilde{\psi}_{i}\left(x_{2}, x_{1}\right)=i e_{i}, & & & 1 \leq i \leq m_{1}, \\
\widetilde{\phi}_{1}\left(x_{2}, e_{1}\right)=-e_{1}, & \widetilde{\phi}_{1}\left(e_{i}, x_{2}\right)=-i e_{i}, & & 1 \leq i \leq m_{1}, \\
\widetilde{\phi}_{2}\left(f_{1}, x_{1}\right)=f_{1}, & \widetilde{\phi}_{3}\left(x_{1}, x_{2}\right)=f_{1}, & \widetilde{\phi}_{4}\left(x_{2}, x_{2}\right)=f_{1} . &
\end{array}
$$


Следствие 3.7. $\operatorname{dim} \overline{B L^{2}\left(R\left(N_{m_{1}}, 1\right), R\left(N_{m_{1}}, 1\right)\right)}=4 m_{1}+7$.

Из утверждений 3.5-3.7 вытекает следующий результат.

Теорема 3.8. $H L^{2}\left(R\left(N_{m_{1}, 1}, 2\right), R\left(N_{m_{1}, 1}, 2\right)\right)=0$.

Докажем равенство $H L^{2}\left(R\left(N_{m_{1}, m_{2}}, 2\right), R\left(N_{m_{1}, m_{2}}, 2\right)\right)=0$ при $m_{2} \geq 2$. Так как в алгебре $R\left(N_{m_{1}, m_{2}}, 2\right)$ не существует такого идеала $J$, что фактор-алгебра $\left.R\left(N_{m_{1}, m_{2}}, 2\right)\right) / J$ изоморфно вкладывается в алгебру Лейбница $R\left(N_{m_{1}, m_{2}}, 2\right)$, вычислим размерность пространств 2-коциклов и 2-кограниц алгебры $R\left(N_{m_{1}, m_{2}}, 2\right)$, посчитав число независимых параметров, задающих произвольные элементы данных пространств.

Предложение 3.9. $\operatorname{dim} \mathrm{ZL}^{2}\left(R\left(N_{m_{1}, m_{2}}, 2\right), R\left(N_{m_{1}, m_{2}}, 2\right)\right)=\left(m_{1}+m_{2}\right)^{2}+$ $4\left(m_{1}+m_{2}\right)+1$.

ДокАЗАтЕЛЬСтво. Пусть $\varphi \in \mathrm{ZL}^{2}\left(R\left(N_{m_{1}, m_{2}}, 2\right), R\left(N_{m_{1}, m_{2}}, 2\right)\right)$. Положим

$$
\begin{aligned}
& \varphi\left(e_{k}, e_{1}\right)=\sum_{i=1}^{m_{1}} \alpha_{1, i}^{k} e_{i}+\sum_{i=1}^{m_{2}} \alpha_{2, i}^{k} f_{i}+\alpha_{k, 1} x_{1}+\alpha_{k, 2} x_{2}, \quad 1 \leq k \leq m_{1}, \\
& \varphi\left(f_{k}, e_{1}\right)=\sum_{i=1}^{m_{1}} \beta_{1, i}^{k} e_{i}+\sum_{i=1}^{m_{2}} \beta_{2, i}^{k} f_{i}+\beta_{k, 1} x_{1}+\beta_{k, 2} x_{2}, \quad 1 \leq k \leq m_{2}, \\
& \varphi\left(x_{1}, e_{1}\right)=\sum_{i=1}^{m_{1}} \delta_{1, i}^{1} e_{i}+\sum_{i=1}^{m_{2}} \delta_{2, i}^{1} f_{i}+\delta_{1}^{1} x_{1}+\delta_{2}^{1} x_{2}, \\
& \varphi\left(e_{1}, x_{1}\right)=\sum_{i=1}^{m_{1}} \delta_{1, i}^{2} e_{i}+\sum_{i=1}^{m_{2}} \delta_{2, i}^{2} f_{i}+\delta_{1}^{2} x_{1}+\delta_{2}^{2} x_{2}, \\
& \varphi\left(x_{2}, e_{1}\right)=\sum_{i=1}^{m_{1}} \delta_{1, i}^{3} e_{i}+\sum_{i=1}^{m_{2}} \delta_{2, i}^{3} f_{i}+\delta_{1}^{3} x_{1}+\delta_{2}^{3} x_{2}, \\
& \varphi\left(e_{1}, x_{2}\right)=\sum_{i=1}^{m_{1}} \delta_{1, i}^{4} e_{i}+\sum_{i=1}^{m_{2}} \delta_{2, i}^{4} f_{i}+\delta_{1}^{4} x_{1}+\delta_{2}^{4} x_{2}, \\
& \varphi\left(e_{1}, f_{1}\right)=\sum_{i=1}^{m_{1}} \alpha_{1, i}^{1,2} e_{i}+\sum_{i=1}^{m_{2}} \alpha_{2, i}^{1,2} f_{i}+\alpha_{1}^{1,2} x_{1}+\alpha_{2}^{1,2} x_{2}, \\
& \varphi\left(f_{1}, f_{1}\right)=\sum_{i=1}^{m_{1}} \alpha_{1, i}^{2,2} e_{i}+\sum_{i=1}^{m_{2}} \alpha_{2, i}^{2,2} f_{i}+\alpha_{1}^{2,2} x_{1}+\alpha_{2}^{2,2} x_{2}, \\
& \varphi\left(x_{i}, x_{j}\right)=\sum_{t=1}^{m_{1}} \gamma_{1, t}^{i, j} e_{t}+\sum_{t=1}^{m_{2}} \gamma_{2, t}^{i, j} f_{t}+\gamma_{1}^{i, j} x_{1}+\gamma_{2}^{i, j} x_{2}, \quad 1 \leq i, j \leq 2, \\
& \varphi\left(x_{t}, f_{1}\right)=\sum_{i=1}^{m_{1}} \xi_{1, i}^{t} e_{i}+\sum_{i=1}^{m_{2}} \xi_{2, i}^{t} f_{i}+\xi_{1}^{t} x_{1}+\xi_{2}^{t} x_{2}, \quad 1 \leq t \leq 2, \\
& \varphi\left(f_{1}, x_{1}\right)=\sum_{i=1}^{m_{1}} \xi_{1, i}^{3} e_{i}+\sum_{i=1}^{m_{2}} \xi_{2, i}^{3} f_{i}+\xi_{1}^{3} x_{1}+\xi_{2}^{3} x_{2}, \\
& \varphi\left(f_{1}, x_{2}\right)=\sum_{i=1}^{m_{1}} \xi_{1, i}^{4} e_{i}+\sum_{i=1}^{m_{2}} \xi_{2, i}^{4} f_{i}+\xi_{1}^{4} x_{1}+\xi_{2}^{4} x_{2} .
\end{aligned}
$$


Подставляя в тождество

$$
\begin{aligned}
\left(d^{2} \varphi\right)(x, y, z)=[x, \varphi(y, z)]-[\varphi(x, y), z] & +[\varphi(x, z), y] \\
& +\varphi(x,[y, z])-\varphi([x, y], z)+\varphi([x, z], y)=0
\end{aligned}
$$

различные элементы $x, y, z \in R\left(N_{m_{1}, m_{2}}, 2\right)$, получаем ограничения на параметры, задающие 2-коцикл $\varphi$.

Из равенства $\left(d^{2} \varphi\right)\left(e_{i}, e_{1}, e_{1}\right)=0$ получим

$$
\varphi\left(e_{i}, e_{2}\right)=-i \alpha_{1,1} e_{1}-\alpha_{1,1}^{1} e_{i+1}, \quad 1 \leq i \leq m_{1} .
$$

Из равенств $\left(d^{2} \varphi\right)\left(e_{i}, e_{1}, e_{j}\right)=0$ и $\left(d^{2} \varphi\right)\left(e_{1}, e_{i}, e_{j}\right)=0$ при $1 \leq i \leq m_{1}, 2 \leq j \leq m_{1}$ вытекает

$$
\varphi\left(e_{i}, e_{j}\right)=-i \alpha_{j-1,1} e_{i}+\left(\alpha_{j-2,1}-\alpha_{1,1}^{j-1}\right) e_{i+1}, \quad 1 \leq i \leq m_{1}, 3 \leq j \leq m_{1} .
$$

Рассмотрев равенство $\left(d^{2} \varphi\right)\left(f_{i}, e_{j}, e_{1}\right)=0$ для $1 \leq i \leq m_{2}, 1 \leq j \leq m_{1}$, заключаeм

$$
\varphi\left(f_{i}, e_{2}\right)=-\left((i-1) \alpha_{1,1}+\alpha_{1,2}\right) f_{i}-\alpha_{1,1}^{1} f_{i+1}, \quad 1 \leq i \leq m_{2},
$$

$\varphi\left(f_{i}, e_{j}\right)=-\left((i-1) \alpha_{j-1,1}+\alpha_{j-1,2}\right) f_{i}+\left(\alpha_{j-2,1}-\alpha_{1,1}^{j-1}\right) f_{i+1}, 1 \leq i \leq m_{2}, 3 \leq j \leq m_{1}$.

Подставляя в тождество $\left(d^{2} \varphi\right)(x, y, z)=0$ различные элементы $x, y, z \in L$, получаем ограничения на параметры, задающие $\varphi$, где слева в импликациях стоят равенства, справа - ограничения:

$$
\begin{aligned}
& \left(d^{2} \varphi\right)\left(x_{1}, f_{1}, x_{1}\right)=0 \Rightarrow \xi_{1,1}^{3}=-\xi_{1,1}^{1}, \xi_{1, i}^{1}=0,2 \leq i \leq m_{1}, \quad \xi_{2, j}^{1}=0,2 \leq j \leq m_{2}, \\
& \left(d^{2} \varphi\right)\left(x_{1}, e_{1}, f_{1}\right)=0 \Rightarrow \xi_{1}^{1}=\alpha_{2,1}^{1,2}=\alpha_{1}^{1,2}=\alpha_{2}^{1,2}=0, \quad \alpha_{1, i}^{1,2}=0,3 \leq i \leq m_{1}, \\
& \xi_{1,1}^{1}=-\alpha_{1,2}^{1,2}, \quad \xi_{2,1}^{1}=-\alpha_{2,2}^{1,2}, \alpha_{2, i}^{1,2}=0, \quad 3 \leq i \leq m_{2}, \\
& \left(d^{2} \varphi\right)\left(e_{1}, x_{2}, f_{1}\right)=0 \Rightarrow \xi_{1,1}^{2}=\xi_{1}^{2}=\alpha_{2,2}^{1,2}=0 \text {, } \\
& \left(d^{2} \varphi\right)\left(f_{1}, f_{1}, x_{1}\right)=0 \Rightarrow \alpha_{2,2}^{2,2}=\alpha_{1,2}^{1,2}, \quad \xi_{2}^{3}=\alpha_{1, i}^{2,2}=0,1 \leq i \leq m_{1}, \\
& \alpha_{2, j}^{2,2}=0,3 \leq j \leq m_{2}, \\
& \left(d^{2} \varphi\right)\left(f_{1}, x_{1}, f_{1}\right)=0 \Rightarrow \xi_{2}^{1}=0 \text {, } \\
& \left(d^{2} \varphi\right)\left(e_{1}, x_{1}, x_{1}\right)=0 \Rightarrow \gamma_{1,1}^{1,1}=\gamma_{1}^{1,1}=0 \\
& \left(d^{2} \varphi\right)\left(f_{1}, x_{1}, x_{1}\right)=0 \Rightarrow \gamma_{2}^{1,1}=0 \\
& \left(d^{2} \varphi\right)\left(x_{1}, e_{1}, x_{1}\right)=0 \Rightarrow \delta_{1,2}^{2}=\delta_{1,2}^{1}, \delta_{2,2}^{2}=0, \gamma_{1, i}^{1,1}=i \delta_{1, i+1}^{1}-\delta_{1, i+1}^{2}, 1 \leq i \leq m_{1}-1 \text {, } \\
& \delta_{2,1}^{2}=-\delta_{2,1}^{1}, \quad \delta_{2}^{2}=-\delta_{2}^{1} \text {, } \\
& \delta_{1}^{2}=-\delta_{1}^{1}, \quad \gamma_{2, i}^{1,1}=(i-1) \delta_{2, i+1}^{1}-\delta_{2, i+1}^{2}, \quad 2 \leq i \leq m_{2}-1, \\
& \left(d^{2} \varphi\right)\left(x_{1}, x_{1}, x_{2}\right)=0 \Rightarrow \gamma_{2,1}^{1,1}=\gamma_{1, i}^{1,2}=0, \quad \gamma_{2, m_{2}}^{1,2}=\frac{1}{m_{2}-1} \gamma_{2, m_{2}}^{1,1}, \quad 2 \leq i \leq m_{1}, \\
& \gamma_{2, i}^{1,2}=\delta_{2, i+1}^{1}-\frac{1}{i-1} \delta_{2, i+1}^{2}, \quad 2 \leq i \leq m_{2}-1, \\
& \left(d^{2} \varphi\right)\left(e_{1}, e_{1}, x_{1}\right)=0 \Rightarrow \delta_{1,1}^{2}=-\delta_{1,1}^{1} \text {, } \\
& \left(d^{2} \varphi\right)\left(e_{1}, x_{1}, x_{2}\right)=0 \Rightarrow \delta_{1}^{4}=\delta_{2}^{4}=\gamma_{1}^{1,2}=0, \quad \gamma_{1,1}^{1,2}=-\delta_{1,2}^{4}, \quad \delta_{1, i}^{4}=0, \quad 3 \leq i \leq m_{1} \text {, } \\
& \delta_{2,1}^{4}=\delta_{2,1}^{1}, \quad \delta_{2, i}^{4}=\frac{1}{i-2} \delta_{2, i}^{2}, \quad 3 \leq i \leq m_{2}, \\
& \left(d^{2} \varphi\right)\left(x_{2}, e_{1}, x_{2}\right)=0 \Rightarrow \gamma_{1}^{2,2}=\delta_{2,1}^{3}=\gamma_{1, i}^{2,2}=0, \quad 1 \leq i \leq m_{1}-1 \text {, }
\end{aligned}
$$




$$
\begin{aligned}
& \gamma_{2, i}^{2,2}=\delta_{2, i+1}^{3}, \quad 1 \leq i \leq m_{2}-1 \\
\left(d^{2} \varphi\right)\left(x_{1}, x_{2}, e_{1}\right)=0 \Rightarrow & \delta_{1,1}^{4}=-\delta_{1,1}^{3}, \quad \gamma_{2,1}^{1,2}=\delta_{2,2}^{1}-\delta_{2,2}^{4}, \\
\left(d^{2} \varphi\right)\left(x_{2}, x_{1}, e_{1}\right)=0 \Rightarrow & \gamma_{1}^{2,1}=\gamma_{2,1}^{2,1}=0, \quad \gamma_{1, i}^{2,1}=i \delta_{1, i+1}^{3}, \quad 1 \leq i \leq m_{1}-1, \\
& \delta_{1}^{3}=\delta_{2}^{3}=0, \quad \gamma_{2, i}^{2,1}=(i-1) \delta_{2, i+1}^{3}, \quad 2 \leq i \leq m_{2}-1, \\
\left(d^{2} \varphi\right)\left(f_{1}, x_{2}, x_{1}\right)=0 \Rightarrow & \gamma_{2}^{2,1}=\xi_{1}^{3}=0, \quad \xi_{1, i}^{4}=-\frac{1}{i} \xi_{1, i}^{3}, \quad 1 \leq i \leq m_{1}, \\
& \delta_{1,2}^{3}=\xi_{2,2}^{4}, \quad \xi_{2, i}^{4}=0, \quad 3 \leq i \leq m_{2}, \\
\left(d^{2} \varphi\right)\left(f_{1}, x_{1}, x_{2}\right)=0 \Rightarrow & \gamma_{2}^{1,2}=0, \quad \delta_{1,2}^{4}=\delta_{1,2}^{3}, \\
\left(d^{2} \varphi\right)\left(e_{1}, f_{1}, x_{2}\right)=0 \Rightarrow & \xi_{1,1}^{3}=\alpha_{1,2}^{1,2}, \quad \xi_{1}^{4}=-\alpha_{1,1}^{1,2} \\
\left(d^{2} \varphi\right)\left(f_{1}, f_{1}, x_{2}\right)=0 \Rightarrow & \xi_{2}^{4}=-\alpha_{2,1}^{2,2}, \quad \alpha_{1}^{2,2}=\alpha_{2}^{2,2}=0 \\
\left(d^{2} \varphi\right)\left(x_{2}, x_{2}, f_{1}\right)=0 \Rightarrow & \xi_{2, i}^{2}=0, \quad 1 \leq i \leq m_{2}, \\
\left(d^{2} \varphi\right)\left(x_{2}, f_{1}, x_{1}\right)=0 \Rightarrow & \xi_{1, i}^{2}=0, \quad 2 \leq i \leq m_{1}, \\
\left(d^{2} \varphi\right)\left(x_{2}, f_{1}, x_{2}\right)=0 \Rightarrow & \xi_{2}^{2}=0, \\
\left(d^{2} \varphi\right)\left(f_{1}, x_{2}, x_{2}\right)=0 \Rightarrow & \gamma_{2}^{2}=\gamma_{1,1}^{2,2}=0 \\
\left(d^{2} \varphi\right)\left(x_{2}, x_{2}, x_{1}\right)=0 \Rightarrow & \gamma_{1, m_{1}}^{2,2}=0, \quad \gamma_{2, m_{2}}^{2,2}=\frac{1}{m_{2}-1} \gamma_{2, m_{2}}^{2,1} .
\end{aligned}
$$

Из равенства $\left(d^{2} \varphi\right)\left(e_{i}, e_{1}, f_{j}\right)=0$ при $1 \leq i \leq m_{1}, 1 \leq j \leq m_{2}$ получим

$$
\begin{gathered}
\varphi\left(e_{i}, f_{1}\right)=i \alpha_{1,1}^{1,2} e_{i}+\alpha_{1,2}^{1,2} e_{i+1}, \varphi\left(e_{i}, f_{2}\right)=-i \beta_{1,1} e_{i}-\left(\beta_{1,1}^{1}+\alpha_{1,1}^{1,2}\right) e_{i+1}, \quad 1 \leq i \leq m_{1}, \\
\varphi\left(e_{i}, f_{j}\right)=-i \beta_{j-1,1} e_{i}+\left(\beta_{j-2,1}-\beta_{1,1}^{j-1}\right) e_{i+1}, \quad 1 \leq i \leq m_{1}, 3 \leq j \leq m_{2} .
\end{gathered}
$$

Из равенств $\left(d^{2} \varphi\right)\left(f_{i}, e_{1}, f_{j}\right)=0$ и $\left(d^{2} \varphi\right)\left(f_{i}, f_{j}, e_{1}\right)=0$ при $1 \leq i, j \leq m_{2}$ следует

$$
\begin{gathered}
\varphi\left(f_{i}, f_{1}\right)=\left((i-1) \alpha_{1,1}^{1,2}+\alpha_{2,1}^{2,2}\right) f_{i}+\alpha_{1,2}^{1,2} f_{i+1}, \\
\varphi\left(f_{i}, f_{2}\right)=-\left((i-1) \beta_{1,1}+\beta_{1,2}\right) f_{i}-\left(\beta_{1,1}^{1}+\alpha_{1,1}^{1,2}\right) f_{i+1}, \\
\varphi\left(f_{i}, f_{j}\right)=-\left((i-1) \beta_{j-1,1}+\beta_{j-1,2}\right) f_{i}+\left(\beta_{j-2,1}-\beta_{1,1}^{j-1}\right) f_{i+1}, \quad 3 \leq j \leq m_{2} .
\end{gathered}
$$

Рассмотрев равенство $\left(d^{2} \varphi\right)(x, y, z)=0$ для следующих троек элементов: $\left\{x_{1}, e_{i}, e_{1}\right\},\left\{e_{i}, e_{1}, x_{1}\right\},\left\{x_{2}, e_{i}, e_{1}\right\},\left\{e_{i}, e_{1}, x_{2}\right\}$ при $1 \leq i \leq m_{1}-1$, последовательно рекуррентным образом получим

$$
\begin{gathered}
\varphi\left(x_{1}, e_{2}\right)=\alpha_{1,1}^{1} e_{1}, \varphi\left(x_{1}, e_{i}\right)=\left(\begin{array}{r}
\left.\alpha_{1,1}^{i-1}-\alpha_{i-2,1}\right) e_{1}, \\
3 \leq i \leq m_{1}, \quad \varphi\left(x_{2}, e_{i}\right)=0,2 \leq i \leq m_{1},
\end{array}\right. \\
\varphi\left(e_{i}, x_{1}\right)=\sum_{t=1}^{i-2}(i-t)\left(\sum_{j=1}^{t} \alpha_{1, j}^{i-t+j-1}-\alpha_{i-t-1,1}\right) e_{t} \\
+\left(\sum_{j=1}^{i-1} \alpha_{1, j}^{j}-\frac{(i+1)(i-2)}{2} \delta_{1}^{1}\right) e_{i-1} \\
-i \delta_{1,1}^{1} e_{i}+\sum_{j=i+1}^{m_{1}}\left(\delta_{j-i+1}^{2}-(j-i) \sum_{t=1}^{i-1} \alpha_{1, j-i+t+1}^{t}\right) e_{j}+\sum_{t=1}^{i-1}\left((i-t+1) \sum_{j=1}^{t} \alpha_{2, j}^{i+j-t-1}\right) f_{t}
\end{gathered}
$$




$$
\begin{aligned}
& +\sum_{j=i}^{m_{2}}\left((i+1-j) \sum_{t=1}^{i-1} \alpha_{2, j-i+t+1}^{t}+\delta_{2, j-i+1}^{2}\right) f_{j}+i\left(\alpha_{i-1,1} x_{1}+\alpha_{i-1,2} x_{2}\right), \quad 2 \leq i \leq m_{1}, \\
& \varphi\left(e_{i}, x_{2}\right)=-i \delta_{1,1}^{3} e_{i}+\delta_{1,2}^{3} e_{i+1}-\sum_{t=1}^{i-1}\left(\sum_{j=1}^{t} \alpha_{2, j}^{i+j-t-1}\right) f_{t}+\left(\delta_{2,1}^{1}-\sum_{t=1}^{i-1} \alpha_{2, t+1}^{t}\right) f_{i} \\
& +\left(\delta_{2,2}^{4}-\sum_{t=1}^{i-1} \alpha_{2, t+2}^{t}\right) f_{i+1}+\sum_{j=i+2}^{m_{2}}\left(\frac{1}{j-i-1} \delta_{2, j-i+1}^{2}-\sum_{t=1}^{i-1} \alpha_{2, j+t-i+1}^{t}\right) f_{j}, 2 \leq i \leq m_{1} .
\end{aligned}
$$

Аналогично, подставляя тройки элементов $\left\{x_{1}, f_{i}, e_{1}\right\},\left\{f_{i}, e_{1}, x_{1}\right\},\left\{f_{i}, e_{1}, x_{2}\right\}$, $\left\{x_{2}, f_{i}, e_{1}\right\}, 1 \leq i \leq m$, в равенство $\left(d^{2} \varphi\right)(x, y, z)=0$, последовательно рекуррентным образом получим

$$
\begin{gathered}
\varphi\left(x_{1}, f_{2}\right)=\left(\alpha_{1,2}^{1,2}+\beta_{1,1}^{1}\right) e_{1}, q \varphi\left(x_{1}, f_{i}\right)=\left(\beta_{1,1}^{i-1}-\beta_{i-2,1}\right) e_{1}, \quad 3 \leq i \leq m_{2}, \\
\varphi\left(f_{i}, x_{1}\right)=\sum_{t=1}^{i-2}(i-t-1)\left(\sum_{j=1}^{t} \beta_{1, j}^{i-t+j-1}-\beta_{i-t-1,1}\right) e_{t}+\left(\alpha_{1,2}^{1,2}-\sum_{t=1}^{i-1} \beta_{1, t+1}^{t}\right) e_{i} \\
+\left(\xi_{1,2}^{3}-2 \sum_{t=1}^{i-1} \beta_{1, t+2}^{t}\right) e_{i+1}-\sum_{j=i+2}^{m_{1}}\left((j-i+1) \sum_{t=1}^{i-1} \beta_{1, j-i+t+1}^{t}\right) e_{j} \\
+\sum_{t=1}^{i-2}\left((i-t) \sum_{j=1}^{t} \beta_{2, j}^{i+j-t-1}\right) f_{t} \\
\left.+\sum_{t=1}^{i-1} \beta_{2, t}^{t}-\frac{(i-2)(i-1)}{2} \delta_{1}^{1}-(i-1) \delta_{2}^{1}\right) f_{i-1}+\left(\xi_{2,1}^{3}-(i-1) \delta_{1,1}^{1}\right) f_{i} \\
\left(\xi_{2, j-i+1}^{3}-(j-i) \sum_{t=1}^{i-1} \beta_{2, j+t-i+1}^{t}\right) f_{j}+(i-1)\left(\beta_{i-1,1} x_{1}+\beta_{i-1,2} x_{2}\right), 2 \leq i \leq m_{2}, \\
\varphi\left(f_{i}, x_{2}\right)=\sum_{j=1}^{i-2}\left(\sum_{t=1}^{j} \beta_{1, t}^{i+t-j-1}-\beta_{i-j-1,1}\right) e_{j}+\left(\sum_{t=1}^{i-1} \beta_{1, t}^{t}+\alpha_{1,1}^{1,2}\right) e_{i-1} \\
+\left(\sum_{t=1}^{i-1} \beta_{1, t+1}^{t}-\alpha_{1,2}^{1,2}\right) e_{i}+\sum_{j=i+1}^{m_{1}}\left(\sum_{t=1}^{i-1} \beta_{1, j+t-i+1}^{t}-\frac{1}{j-i+1} \xi_{1, j-i+1}^{3}\right) e_{j} \\
+\left(\xi_{2,1}^{4}-(i-1) \delta_{1,1}^{3}\right) f_{i}+\xi_{2,2}^{4} f_{i+1}+\beta_{i-1,1} x_{1}+\beta_{i-1,2} x_{2}, \quad 2 \leq i \leq m_{2}, \\
\varphi\left(x_{2}, f_{i}\right)=0, \quad 1 \leq i \leq m_{2} .
\end{gathered}
$$

Равенства $\left(d^{2} \varphi\right)\left(e_{m_{1}}, e_{1}, x_{1}\right)=\left(d^{2} \varphi\right)\left(f_{m_{2}}, e_{1}, x_{1}\right)=\left(d^{2} \varphi\right)\left(f_{m_{2}}, e_{1}, x_{2}\right)=0$ влекут

$$
\begin{gathered}
\alpha_{1,1}^{m_{1}}=\alpha_{m_{1}-1,1}, \quad \alpha_{2,1}^{m_{1}}=\alpha_{m_{1}, 1}=\alpha_{m_{1}, 2}=0 \\
\alpha_{1, i}^{m_{1}}=\alpha_{m_{1}-i, 1}-\sum_{j=1}^{i-1} \alpha_{1, j}^{m_{1}+j-i}, \quad 2 \leq i \leq m_{1}-1, \\
\alpha_{2, i}^{m_{1}}=-\sum_{j=1}^{i-1} \alpha_{2, j}^{m_{1}+j-i}, 2 \leq i \leq m_{2}, \quad \delta_{1}^{1}=\frac{2}{\left(m_{1}+2\right)\left(m_{1}-1\right)} \sum_{i=1}^{m_{1}} \alpha_{1, i}^{i},
\end{gathered}
$$




$$
\begin{gathered}
\beta_{m_{2}, 1}=\beta_{m_{2}, 2}=\beta_{2,1}^{m_{2}}=0, \quad \beta_{1,1}^{m_{2}}=\beta_{m_{2}-1,1}, \quad \beta_{1, i}^{m_{2}}=\beta_{m_{2}-i, 1}-\sum_{j=1}^{i-1} \beta_{1, j}^{m_{2}-i+j}, \\
\beta_{2, i}^{m_{2}}=-\sum_{j=1}^{i-1} \beta_{2, j}^{m_{2}+j-i}, \delta_{2}^{1}=-\frac{m_{2}-1}{\left(m_{1}+2\right)\left(m_{1}-1\right)} \sum_{i=1}^{m_{1}} \alpha_{1, i}^{i}+\frac{1}{m_{2}} \sum_{i=1}^{m_{2}} \beta_{2, i}^{i}, \\
\xi_{1,2}^{3}=2 \sum_{t=1}^{m_{2}} \beta_{1, t+2}^{t}, \quad \alpha_{1,2}^{1,2}=\sum_{t=1}^{m_{2}} \beta_{1, t+1}^{t}, \quad \alpha_{1,1}^{1,2}=-\sum_{t=1}^{m_{2}} \beta_{1, t}^{t}, \quad 2 \leq i \leq m_{2}-1 .
\end{gathered}
$$

В описании произвольного элемента $\varphi \in \mathrm{ZL}^{2}\left(R\left(N_{m_{1}, m_{2}}, 2\right), R\left(N_{m_{1}, m_{2}}, 2\right)\right)$ участвуют $\left(m_{1}+m_{2}\right)^{2}+4\left(m_{1}+m_{2}\right)+1$ штук независимых параметров, следовательно,

$$
\operatorname{dim} \mathrm{ZL}^{2}\left(R\left(N_{m_{1}, m_{2}}, 2\right), R\left(N_{m_{1}, m_{2}}, 2\right)\right)=\left(m_{1}+m_{2}\right)^{2}+4\left(m_{1}+m_{2}\right)+1 .
$$

Из определения пространства $\mathrm{BL}^{2}\left(R\left(N_{m_{1}, m_{2}}, 2\right), R\left(N_{m_{1}, m_{2}}, 2\right)\right)$ и предложения 3.2 при $s=2$ нетрудно заключить, что $\operatorname{dim} \mathrm{BL}^{2}\left(R\left(N_{m_{1}, m_{2}}, 2\right), R\left(N_{m_{1}, m_{2}}, 2\right)\right)$ $=\left(m_{1}+m_{2}+2\right)^{2}-3$.

Из определения второй группы когомологий имеем

$$
H L^{2}\left(R\left(N_{m_{1}, m_{2}}, 2\right), R\left(N_{m_{1}, m_{2}}, 2\right)\right)=0 .
$$

Обобщая процесс доказательства равенства (1), можно доказать следующую теорему.

Теорема 3.10. $H L^{2}\left(R\left(N_{m_{1}, \ldots, m_{s}}, s\right), R\left(N_{m_{1}, \ldots, m_{s}}, s\right)\right)=0$.

ЗАмечАНИЕ 3.11. При вычислении 2-й группы когомологий алгебры $R\left(N_{m_{1}, \ldots, m_{s}}, s\right)$ получено, что размерности пространств 2-коциклов и 2 -кограниц равны $\left(m_{1}+m_{2}+\cdots+m_{s}+s\right)^{2}-(s+1)$.

Следствие 3.12. Алгебра $R\left(N_{m_{1}, \ldots, m_{s}}, s\right)$ является жесткой алгеброй в многообразии алгебр Лейбница размерности $m_{1}+\cdots+m_{s}+s$.

\section{ЛИТЕРАТУРА}

1. Блох А. Об обобщении понятия алгебры Ли // Докл. АН. 1965. Т. 165, № 3. С. 471-473.

2. Loday J.-L. Une version non commutative des algèbres de Lie: les algèbres de Leibniz // Enseign. Math. 1993. V. 39. P. 269-293.

3. Balavoine $D$. Déformations et rigidité géométrique des algebras de Leibniz // Comm. Algebra. 1996. V. 24. P. 1017-1034.

4. Hochschild G., Serre J-P. Cohomology of Lie algebras // Ann. Math. 1953. V. 57. P. 591-603.

5. Ancochea Bermúdez J. M., Campoamor-Stursberg R. Cohomologically rigid solvable Lie algebras with a nilradical of arbitrary characteristic sequence // Linear Algebra Appl. 2016. V. 488. P. $135-147$.

6. Khalkulova Kh. A., Abdurasulov K. K., Solvable Lie algebras with maximal dimension of complementary space to nilradical // Uzbek Math. J. 2018. V. 1. P. 90-98.

7. Ancochea Bermúdez J. M., Campoamor-Stursberg R. On a complete rigid Leibniz non-Lie algebra in arbitrary dimension // Linear Algebra Appl. 2013. V. 438. P. 3397-3407.

Поступила в редакиию 13 марта 2019 г.

После доработки 13 января 2020 г.

Принята к публикации 19 февраля 2020 г.

Мамадалиев Уктамжон Хасанбоевич, Омиров Бахром Абдазович

Национальный университет Узбекистана,

ул. Университетская, 4, Ташкент 100174, Узбекистан

mamadaliyevuktamjon@mail.ru, omirovb@mail.ru 